\title{
ON DIMENSIONALITY OF MULTIPATH FIELDS: SPATIAL EXTENT AND RICHNESS
}

\author{
Haley M. Jones, Rodney A. Kennedy and Thushara D. Abhayapala* \\ Department of Telecommunications Engineering \\ Research School of Information Sciences and Engineering \\ Institute of Advanced Studies \\ The Australian National University \\ ACT 0200, Australia
}

\begin{abstract}
We establish that an arbitrary narrowband multipath field in any circular region in two dimensional space has an intrinsic functional dimensionality of $(\pi e) R / \lambda \approx 8.54 R / \lambda$ that scales only linearly with radius $R / \lambda$ in wavelengths. This result implies there is no such thing as an arbitrarily complicated multipath field. That is, a field generated by any number of nearfield and farfield, specular and diffuse multipath reflections is no more complicated than a field generated by a limited number plane waves. As such, there are limits on how rich multipath can be. This result has significant implications including means: i) to determine a parsimonious parameterization for arbitrary multipath fields, ii) of synthesizing arbitrary multipath fields with arbitrarily located nearfield or farfield, spatially discrete or continuous sources. We give examples of multipath field analysis and synthesis.
\end{abstract}

\section{INTRODUCTION}

The study of the spatial aspects of multipath in wireless communications environments is an increasingly important addition to the study of the temporal aspects in the search for ways to improve system performance, including range extension, capacity improvement, high data rates and improved bit error rate performance $[1,2]$. The understanding of the spatial characteristics of wireless channels is incomplete and the true potential of spacetime receivers has yet to be realized in a form that is compatible with the restrictions imposed by physics.

There are many approaches to characterizing the spatial aspects of the wireless multipath environment in the literature. A common approach is to assume a multiple discrete farfield source model $[3,4]$. The complex gains of the multipaths are linearly combined at each receiver sensor, weighted by the gain of that sensor in the direction of arrival of the corresponding multipath component. The complex gains of the multipaths are modelled according to the assumed distribution of scatterers causing the multipath.

In experimental approaches, physical measurements are taken in order to statistically characterize a specific wireless multipath environment with the intent of generalizing for similar environments. Parameter characteristics for which a statistical model can

T.D. Abhayapala also has a joint appointment with the Faculty of Engineering \& Information Technology, ANU.

This work has been supported by an Australian Research Council SPIRT grant and NEC Australia. be useful include angle of arrival [5], spatial signal correlation [6] and changes in multipath profile [7]. In yet other approaches, geometrical models are used to characterize diffuse multipath fields [8] and, in [9], a purely theoretical model is used in which the concept of multipath shaping factors is introduced to derive second order small-scale fading statistics.

We use a theoretical model which can be applied to any narrowband multipath environment regardless of the number or nature of the multipath sources. We show that there is an intrinsic dimensionality to a narrowband multipath field in a region of space of a given size. We use this to show that there is an upper limit on the degree of multipath richness which can exist in a given sized area. Closely matched theoretical and experimental results are presented.

We show that the field in a given area of space can be represented by relatively few terms of a functional expansion, the number of which represent the dimensionality. Furthermore, it is possible to synthesize an arbitrary field as accurately as desired using an appropriate combination of arbitrary sources, nearfield or farfield, discrete or continuous. We demonstrate the specific case of a small number of plane wave sources synthesizing an arbitrary field. Such small parameterizations of arbitrary narrowband multipath fields indicate that even a diffuse field can be synthesized by relatively few discrete multipath terms.

\section{GENERAL 2D MULTIPATH FIELD}

Consider a two dimensional (2D) narrowband multipath interference in a given sized region ${ }^{1}$. The multipath signals may have sources which are nearfield or farfield, specular or diffuse. We use polar coordinates to represent a point in space $\boldsymbol{x} \equiv\left(\|\boldsymbol{x}\|, \phi_{x}\right)$. The field, $F(\boldsymbol{x} ; k)$, is a function of the position and the wave number, $k=2 \pi / \lambda$, where $\lambda$ is the wavelength. It is a solution to the Helmholtz wave equation in polar coordinates [10], the most general solution of which is

$$
F(\boldsymbol{x} ; k)=\sum_{n=-\infty}^{\infty} \alpha_{n} J_{n}(k\|\boldsymbol{x}\|) e^{i n \phi_{x}}
$$

where $\alpha_{n}$ are complex constants independent of position and $J_{n}(\cdot)$ is the order $n$ Bessel function [11]. That is, in (1) the field strength

\footnotetext{
${ }^{1}$ This models the situation in 3D where the multipath is restricted to the horizontal plane, having no components arriving at significant elevations. As such, the multipath field is height invariant.
} 
at a point $\boldsymbol{x}$ is represented as a weighted sum of orthogonal basis functions.

Plane and circular waves are examples of 2D waves which can be expressed in the form (1). A single plane wave with complex amplitude $a_{p}$ and propagation direction $\phi_{p}$ has $\alpha_{n}=a_{p} i^{n} e^{-i n \phi_{p}}$ [12, p.66]. A single circular wave with source position vector, $\boldsymbol{y}_{p} \equiv\left(\left\|\boldsymbol{y}_{p}\right\|, \phi_{p}\right)$, has $\alpha_{n}=a_{p} e^{-i n \phi_{p}} H_{n}^{(1)}\left(k\left\|\boldsymbol{y}_{p}\right\|\right)[12, \mathrm{p} .66]$ where $H_{n}^{(1)}(\cdot)$ is the order $n$ Hankel function of the first kind.

We illustrate an application of (1) with fields generated by a superposition of $P$ plane waves. Let plane wave of index $p$ have complex amplitude $a_{p}$ and propagation direction $\phi_{p}$ with normalized direction $\hat{\boldsymbol{\eta}}_{p} \equiv\left(\cos \phi_{p}, \sin \phi_{p}\right)^{\prime}$. The field strength at $\boldsymbol{x}$ is then given by

$$
\begin{aligned}
F(\boldsymbol{x} ; k) & =\sum_{p=1}^{P} a_{p} e^{-i k\left\langle\boldsymbol{x}, \hat{\boldsymbol{\eta}}_{p}\right\rangle} \\
& =\sum_{p=1}^{P} a_{p} \sum_{n=-\infty}^{\infty} i^{n} J_{n}(k\|\boldsymbol{x}\|) e^{i n\left(\phi_{x}-\phi_{p}\right)}
\end{aligned}
$$

where (2) is a cartesian form and (3) is the polar equivalent. Note (3) is in the form of (1) with

$$
\alpha_{n}=\sum_{p=1}^{P} a_{p} i^{n} e^{-i n \phi_{p}}=\sum_{p=1}^{P} a_{p} e^{-i n\left(\phi_{p}-\pi / 2\right)} .
$$

Similarly, a field of $P$ circular waves, where $\boldsymbol{y}_{p} \equiv\left(\left\|\boldsymbol{y}_{p}\right\|, \phi_{p}\right)$ is the position vector of the source of index $p$, is given by

$$
\begin{aligned}
F(\boldsymbol{x} ; k) & =\sum_{p=1}^{P} a_{p} \frac{e^{i k\left\|\boldsymbol{x}-\boldsymbol{y}_{p}\right\|}}{\left\|\boldsymbol{x}-\boldsymbol{y}_{p}\right\|} \\
& =\sum_{p=1}^{P} a_{p} \sum_{n=-\infty}^{\infty} H_{n}^{(1)}\left(k\left\|\boldsymbol{y}_{p}\right\|\right) J_{n}(k\|\boldsymbol{x}\|) e^{i n\left(\phi_{x}-\phi_{p}\right)}
\end{aligned}
$$

and for the representation (1) we have

$$
\alpha_{n}=\sum_{p=1}^{P} a_{p} H_{n}^{(1)}\left(k\left\|\boldsymbol{y}_{p}\right\|\right) e^{-i n \phi_{p}} .
$$

We could also form a field of plane and circular waves by linearly combining (3) and (6) to obtain an equation of the form of (1), with $\alpha_{n}$ the weighted sum of (4) and (7).

The Bessel functions $J_{n}(\cdot)$ for $n \geq 1$ in (1) have a spatial high pass character $\left(J_{0}(\cdot)\right.$ is spatially low pass). That is, as illustrated in Fig. 1 , for $n=8$ and $n=80, J_{n}(z)$ starts small increasing monotonically to its maximum at arguments around $O(n)$ before decaying asymptotically to zero as $z \rightarrow \infty$ (oscillating as it does so). Also shown in Fig. 1 are limits imposed by three upper bounds on $\left|J_{n}(z)\right|: 1 / n !(z / 2)^{n}$ (see Appendix A), $0.6748851 / n^{1 / 3}$ and $0.7857468704 / z^{1 / 3}[13]$.

\section{DIMENSIONALITY OF MULTIPATH}

We wish to quantify the complexity of an arbitrary multipath field $F(\boldsymbol{x} ; k)$ in circular region of radius $R / \lambda$ in wavelengths by defining the effective dimensionality of the field. We do this by truncating the series in (1) and determining the minimum number of terms, $2 N+1$, for the field $F_{N}(\boldsymbol{x} ; k)$, so generated, to be within
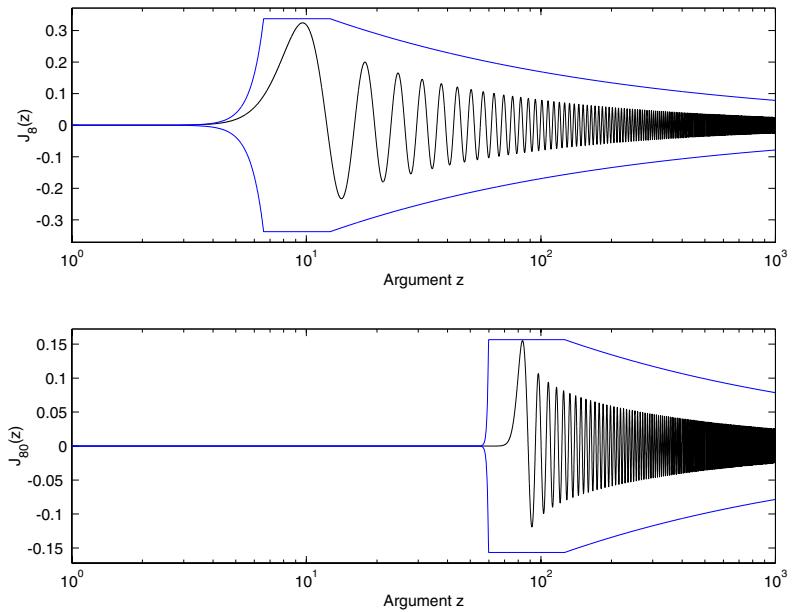

Fig. 1. High pass character of the Bessel functions $J_{8}(z)$ and $J_{80}(z)$ versus argument $z$ (logarithmic scale). Also shown are a combination of three bounds as described in the text.

a specified error, $\varepsilon$, of the actual field $F(\boldsymbol{x} ; k)$. Thus, the approximate field strength $F_{N}(\boldsymbol{x} ; k)$ is defined by the finite sum

$$
F_{N}(\boldsymbol{x} ; k)=\sum_{n=-N}^{N} \alpha_{n} J_{n}(k\|\boldsymbol{x}\|) e^{i n \phi_{x}},
$$

where we wish the approximation to be sufficiently accurate for $\|\boldsymbol{x}\| \leq R$.

Fig. 2 shows the actual field strength of the sum of 30 plane waves, as in (2), over a $3 \lambda \times 3 \lambda$ area, compared with the same field represented by the truncated series in (8) with $\alpha_{n}$ given by (4) and $N=7$. Clearly $F_{N}(\boldsymbol{x} ; k)$ can model very well $F(\boldsymbol{x} ; k)$ in a finite region about the origin.
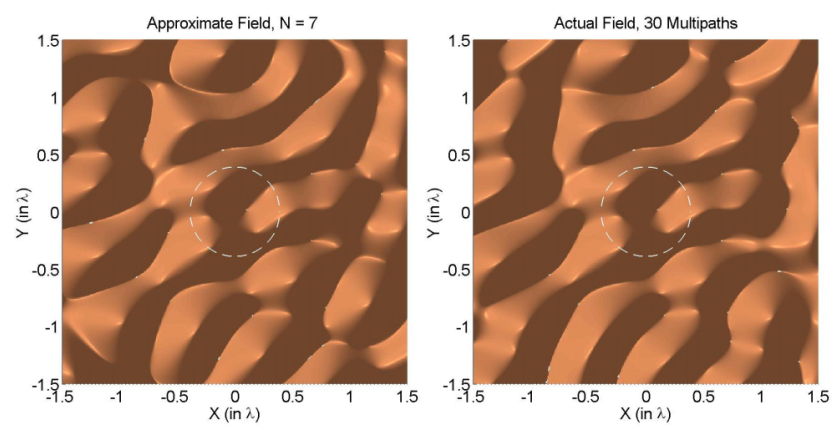

Fig. 2. Example of accuracy of truncation in (8). The actual field (2) has 30 component plane waves in random directions. The approximate field (8) has $2 N+1=15$ plane waves. The approximate field is within $1 \times 10^{-3}$ of the actual field for $R / \lambda \leq 0.3890$.

\subsection{Bounding the Relative Error}

To form a relative error, first bound the peak amplitude of the multipath field to unity. This implies, by Appendix A, that $\left|\alpha_{n}\right| \leq$ $1, \forall n$. Define the error between the actual and approximate fields 
by $\varepsilon_{N}(\boldsymbol{x})$. Then

$$
\begin{aligned}
\varepsilon_{N}(\boldsymbol{x}) & =\left|F(\boldsymbol{x} ; k)-F_{N}(\boldsymbol{x} ; k)\right|=\left|\sum_{|n|>N} \alpha_{n} J_{n}(k\|\boldsymbol{x}\|) e^{i n \phi_{x}}\right| \\
& \leq \sum_{|n|>N}\left|J_{n}(k\|\boldsymbol{x}\|)\right|=2 \sum_{n>N}\left|J_{n}(k\|\boldsymbol{x}\|)\right|
\end{aligned}
$$

Now we need a bound on $\left|J_{n}(\cdot)\right|$ for $n>N$. For integer $n \geq 0$, the order $n$ Bessel function is given by [14]

$$
J_{n}(z)=\sum_{\ell=0}^{\infty} \frac{(-1)^{\ell}(z)^{2 \ell+n}}{2^{2 \ell+n} \ell !(\ell+n) !}, \quad n \geq 0 .
$$

It is shown in Appendix B that $J_{n}(\cdot)$ is always bounded by the first term in (10). An example of this upper bound is shown in Fig. 1 for $J_{8}(\cdot)$ and $J_{80}(\cdot)$.

By using the Stirling lower bound on $n$ ! [14] we can further bound $\left|J_{n}(k\|\boldsymbol{x}\|)\right|$, as follows

$$
\begin{aligned}
\left|J_{n}(k\|\boldsymbol{x}\|)\right| & \leq \frac{(k\|\boldsymbol{x}\|)^{n}}{2^{n} n !} \leq \frac{1}{\sqrt{2 \pi n}}\left(\frac{k e\|\boldsymbol{x}\|}{2 n}\right)^{n} \\
& \leq \frac{\rho(N, R)^{n}}{\sqrt{2 \pi(N+1)}}, \quad n>N,\|\boldsymbol{x}\| \leq R .
\end{aligned}
$$

where

$$
\rho(N, R)=\frac{k e R}{2(N+1)}=\frac{\pi e R / \lambda}{(N+1)} .
$$

Substituting (11) and (12) into (9) and choosing $N$ large enough such that $\rho(N, R)<1$, we obtain an expression for a bound on the error for given values of $N$ and $R$

$$
\varepsilon_{N}(\boldsymbol{x}) \leq \sqrt{\frac{2}{(N+1) \pi}} \cdot \frac{\rho(N, R)^{N+1}}{1-\rho(N, R)}, \quad \forall\|\boldsymbol{x}\| \leq R .
$$

The restriction on $\rho(N, R)$ gives a lower bound on $N$

$$
N>(\pi e) R / \lambda-1 \approx 8.54 R / \lambda .
$$

With $N$ thus selected $F(\boldsymbol{x} ; k)$ and $F_{N}(\boldsymbol{x} ; k)$ are essentially indistinguishable within $\|\boldsymbol{x}\| \leq R$. As $2 N+1$ basis elements are used then $2 N+1$ characterizes the dimensionality.

The dimensionality of fields of radius $R / \lambda \leq 3$, for error thresholds between $10^{-1}$ and $10^{-4}$ are shown in Fig. 3. The number of extra terms required to achieve an error of $10^{-4}$ over an error of $10^{-1}$ is relatively small. For $R / \lambda=1$, the minimum values of $N$ required are 10 and 15, respectively, meaning that just 10 extra terms give 3 orders of magnitude improvement in accuracy.

Simulation results for the relationship between $N$ and the error $\varepsilon$ between actual and approximate fields of radius $R / \lambda=1$ are shown in Fig. 4. Simulated actual fields had from $P=4$ to 50 plane wave components. The error appears to converge, regardless of $P$ at around $N=10$.

\section{Remarks}

1. Field complexity or dimensionality, $(2 N+1)$, increases linearly with $R / \lambda$.

2. The actual number of terms required to represent an arbitrary field to high accuracy is relatively small. The addition of just a few terms can give orders of magnitude improvement in the relative error.

3. Any wave field, including a a diffuse field, consisting of any number of actual wave components may be represented by relatively few parameters.

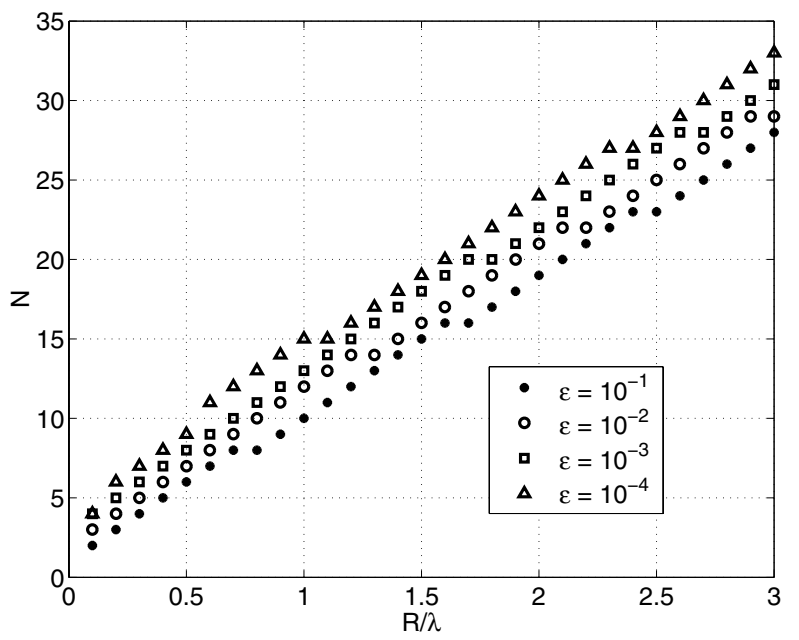

Fig. 3. Minimum $N$ required for different error thresholds $\varepsilon$, as given by theoretical bounds (13) and (14), for increasing values of radius $R / \lambda$.

\section{PLANE WAVE SYNTHESIS}

We have shown in (8) that an arbitrary wave field in a given region can be represented by a finite number of terms, regardless of the complexity of the scattering environment. We now show that such a field can also be closely approximated by an appropriate combination of plane waves.

Once the coefficients $\alpha_{n}$ in (8) are determined for the field of interest, one can use (4) to define a set of plane waves producing the same $\alpha_{n}$ 's over the critical indices $|n| \leq N$. Let $\boldsymbol{\alpha}=\left[\alpha_{-N}, \cdots, \alpha_{n}, \cdots, \alpha_{N}\right]^{\prime}$ and $\boldsymbol{a}=\left[a_{1}, \cdots, a_{p}, \cdots, a_{P}\right]^{\prime}$. We define the diagonal matrix $\Theta=\operatorname{diag}\left[i^{N}, \cdots, i^{n}, \cdots, i^{-N}\right]$ and the Vandermonde matrix

$$
\boldsymbol{V}=\left[\begin{array}{ccccc}
e^{i N \phi_{1}} & \cdots & e^{i N \phi_{p}} & \cdots & e^{i N \phi_{P}} \\
\vdots & \vdots & \vdots & \vdots & \vdots \\
e^{-i n \phi_{1}} & \cdots & e^{-i n \phi_{p}} & \cdots & e^{-i n \phi_{P}} \\
\vdots & \vdots & \vdots & \vdots & \vdots \\
e^{-i N \phi_{1}} & \cdots & e^{-i N \phi_{p}} & \cdots & e^{-i N \phi_{P}}
\end{array}\right]
$$

Then we can rewrite (4) in matrix form as

$$
\Theta \alpha=V a .
$$

As each of the $\phi_{p}$ 's are distinct, it is known that $\boldsymbol{V}$ is non-singular. Thus, given specific $\boldsymbol{\alpha}, \boldsymbol{V}$ and $\Theta$ we can always solve (16) for a. Therefore, at most $P=2 N+1$ plane waves from arbitrary directions can synthesize an arbitrary field over a region $\|\boldsymbol{x}\| \leq R$ whenever $N \approx(\pi e) R / \lambda$.

\section{Remarks}

1. There are an infinite number of plane wave combinations which can represent a given field $F(\boldsymbol{x} ; k)$. The choice of directions $\left\{\phi_{p}\right\}$ is arbitrary.

2. By choosing $\phi_{p}=2 p \pi / P, \boldsymbol{V}$ in (15) becomes a scaled discrete Fourier transform matrix and the plane wave $a_{p}$ can be computed using the FFT from the weights $\alpha_{n}$. 


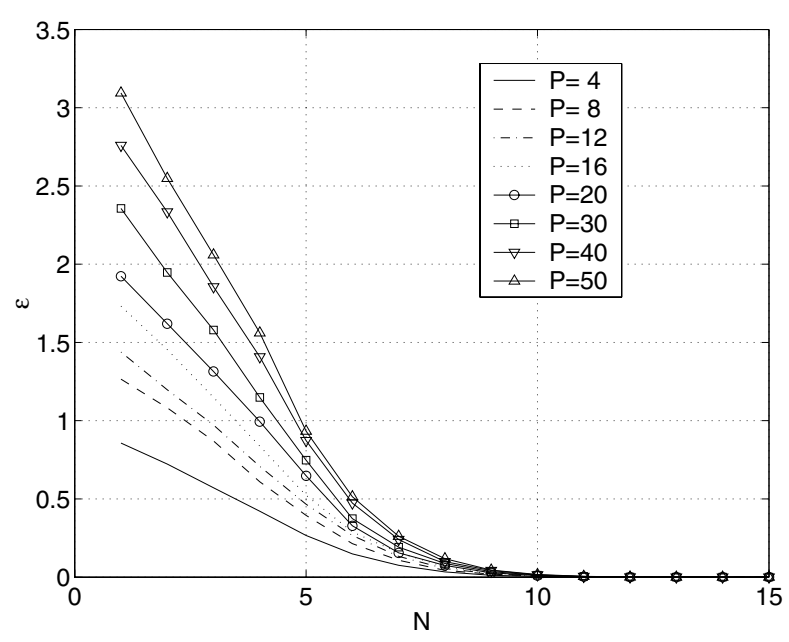

Fig. 4. Average error between actual and approximate fields, as in (2) and (8) for up to $P=50$ plane waves and $N \leq 15$ for $R / \lambda=1$.

3. The result is not restricted to plane waves, any superposition of sources can be used, such as a superposition of nearfield point sources, etc. That is, $2 N+1$ distinct sources generically generate a set of distinct and linearly independent $\boldsymbol{\alpha}$ 's which form a basis.

4. Wave field synthesis reduces to a problem in linear algebra implying that $2 N+1$ sources are necessary, in general.

\section{CONCLUSIONS}

We have defined and determined the dimensionality of a wave field as a measure of field complexity. The dimensionality increases only linearly with the radius of the field. For example, we have shown that an arbitrary narrowband wave field can be reproduced as accurately as desired by a limited number of appropriately weighted plane waves of arbitrary direction.

The existence of small dimensional parameterizations of arbitrary narrowband multipath fields indicate that even a diffuse field can be synthesized by relatively few discrete multipath terms.

All results have been extended to the three-dimensional and broadband cases and will be reported separately.

\section{A. BOUNDEDNESS OF $\left|\alpha_{n}\right|$}

Consider the magnitudes of the $\alpha_{n}$ coefficients for a superposition of a possibly infinite number of plane waves indexed by $p$ with amplitudes $a_{p}$. From (4)

$$
\left|\alpha_{n}\right| \leq\left|\sum_{p} a_{p} i^{n} e^{-i n\left(\phi_{p}-\frac{\pi}{2}\right)}\right| \leq \sum_{p}\left|a_{p}\right|
$$

The RHS of (17) is an upper bound on the field strength at any point being the sum of the amplitudes of the plane waves constituting the given wave field. On physical grounds we assume the field is bounded at all points in space which implies $\sum_{p}\left|a_{p}\right|<B$. Without loss of generality we take $B=1$, amounting to a normalization such that the field strength is is bounded by unity. Hence $\left|\alpha_{n}\right| \leq 1 \forall n$.

\section{B. UPPER BOUND ON $J_{n}(\cdot)$}

From [11, p.192] we have, for $n>-1 / 2$

$$
\begin{aligned}
J_{n}(z) & =\frac{1}{\sqrt{\pi} \Gamma(n+1 / 2)}\left(\frac{z}{2}\right)^{n} \int_{-1}^{+1} e^{i \lambda z}\left(1-\lambda^{2}\right)^{n-1 / 2} d \lambda \\
& \leq \frac{1}{\sqrt{\pi} \Gamma(n+1 / 2)}\left(\frac{z}{2}\right)^{n} \int_{-1}^{+1}\left(1-\lambda^{2}\right)^{n-1 / 2} d \lambda
\end{aligned}
$$

where $\Gamma(\cdot)$ is the Gamma function. From [15, eqn. 15.24], the integral in (18) is given by $\sqrt{\pi} \Gamma(n+1 / 2) / \Gamma(n+1)$. QED

\section{REFERENCES}

[1] A. J. Paulraj and C. B. Papadias, "Space-Time Processing for Wireless Communications," IEEE SP Mag., vol. 14, no. 5, pp. 49-83, Nov 1997.

[2] R. Kohno, "Spatial and Temporal Communication Theory Using Adaptive Antenna Array," IEEE Pers. Com., vol. 5, no. 1, pp. 28-35, Feb 1998.

[3] X. Wang and H. V. Poor, "Space-Time Multiuser Detection in Multipath CDMA Channels," IEEE Tr. SP, vol. 47, no. 9, pp. 2356-2374, Sep 1999.

[4] G. G. Raleigh and J. M. Cioffi, "Spatio-Temporal Coding for Wireless Communication," IEEE Tr. Com., vol. 46, no. 3, pp. 357-366, Mar 1998.

[5] Q. H. Spencer, B. D. Jeffs, M. A. Jensen, and A. L. Swindlehurst, "Modeling the Statistical Time and Angle of Arrival Characteristics of an Indoor Multipath Channel," IEEE J.SAC, vol. 18, no. 3, pp. 347-360, Mar 2000.

[6] P. C. Eggers, J. Toftgard, and A. M. Oprea, "Antenna Systems for Base Station Diversity in Urban Small and Micro Cells," IEEE J.SAC, vol. 11, no. 7, pp. 1046-1057, Sep 1999.

[7] S.-S. Jeng, G. Xu, H.-P. Lin, and W. J. Vogel, "Experimental Studies of Spatial Signature Variation at $900 \mathrm{MHz}$ for Smart Antenna Systems," IEEE Tr. AP, vol. 46, no. 7, pp. 953-962, Jul 1998.

[8] O. Norklit and J. B. Anderson, "Diffuse Channel Model and Experimental Results for Array Antennas in Mobile Environments," IEEE Tr. AP, vol. 46, no. 6, pp. 834-840, Jun 1998.

[9] G. D. Durgin and T. S. Rappaport, "Theory of Multipath Shape Factors for Small-Scale Fading Wireless Channels," IEEE Tr. AP, vol. 48, no. 5, pp. 682-692, May 2000.

[10] C. A. Coulson and A. Jeffrey, Waves: A mathematical approach to the common types of wave motion, Longman, London, second edition, 1977.

[11] N. W. McLachlan, Bessel Functions for Engineers, Oxford University Press, London, second edition, 1961.

[12] D. Colton and R. Kress, Inverse Acoustic and Electromagnetic Scattering Theory, Springer-Verlag, 1992.

[13] L. Landau, "Bessel Functions: Monotonicity and Bounds," Journal of the London Mathematical Society, vol. 61, no. 1, pp. 197-215, Feb 2000.

[14] E. Kreyszig, Advanced Engineering Mathematics, John Wiley \& Sons, New York, 1988.

[15] M. R. Spiegel, Mathematical Handbook of Formulas and Tables, McGraw Hill, 1968. 